\title{
Insertion of electrode array using percutaneous cochlear implantation technique: a cadaveric study
}

\author{
Ramya Balachandran $^{1}$, Jason E. Mitchell ${ }^{2}$, Jack Noble ${ }^{3}$, Daniel Schurzig ${ }^{1}$, Grégoire Blachon ${ }^{1}$, \\ Theodore R. McRackan ${ }^{1}$, Robert J. Webster ${ }^{2}$, Benoit M. Dawant ${ }^{3}$, J. Michael Fitzpatrick ${ }^{3}$, \\ Robert F. Labadie ${ }^{1}$ \\ Dept. of ${ }^{1}$ Otolaryngology, ${ }^{2}$ Mechanical Engineering, ${ }^{3}$ Electrical Engineering \& Computer Science \\ Vanderbilt University, Nashville, TN 37212 \\ Email: ramya.balachandran@vanderbilt.edu
}

\begin{abstract}
Cochlear implantation is a surgical procedure for treating patients with hearing loss in which an electrode array is inserted into the cochlea. The traditional surgical approach requires drilling away a large portion of the bone behind the ear to provide anatomical reference and access to the cochlea. A minimally-invasive technique, called percutaneous cochlear implantation (PCI), has been proposed that involves drilling a linear path from the lateral skull to the cochlea avoiding vital structures and inserting the implant using that drilled path. The steps required to achieve PCI safely include: placing three bone-implanted markers surrounding the ear, obtaining a CT scan, planning a surgical path to the cochlea avoiding vital anatomy, designing and constructing a microstereotactic frame that mounts on the markers and constrains the drill to the planned path, affixing the frame on the markers, using it to drill to the cochlea, and inserting the electrode through the drilled path. We present in this paper a cadaveric study demonstrating the PCI technique on three temporal bone cadaveric specimens for inserting electrode array into the cochlea. A custom fixture, called a Microtable, which is a type of microstereotactic frame that can be constructed in less than five minutes, was fabricated for each specimen and used to reach the cochlea. The insertion was successfully performed on all three specimens. Postinsertion CT scans confirm the correct placement of the electrodes inside the cochlea without any damage to the facial nerve.
\end{abstract}

Keywords: Percutaneous cochlear implantation, microstereotactic frame, minimally-invasive surgery.

\section{INTRODUCTION}

Cochlear implantation is a surgical treatment for patients with sensorineural hearing loss. An electrode array is inserted into the cochlea to stimulate the auditory nerve and is coupled to an internal receiver, which forwards signals to the electrode array. The current surgical technique for cochlear implantation includes mastoidectomy and posterior

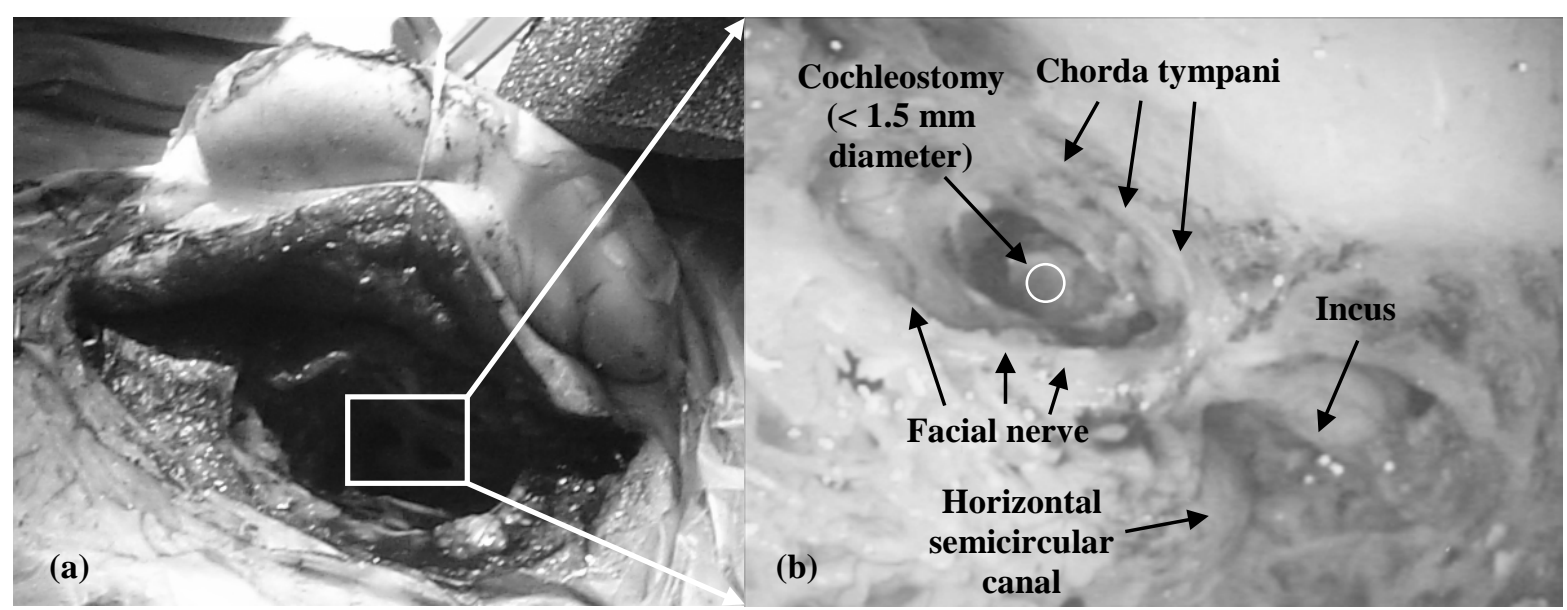

Figure 1. (a) Typical drilled mastoid during a traditional cochlear implant surgery. (b) Magnified view of the facial recess region showing the vital structures. 
tympanotomy, which requires a wide surgical exposure to clearly identify anatomic landmarks for avoiding injury to critical structures [1] such as the facial nerve, which when damaged causes paralysis of the ipsilateral side of the face, and the chorda tympani, which when damaged causes loss of sensitivity of the ipsilateral tongue tip. Figure 1 shows the wide exposure that is typically required during a cochlear implant surgery and a magnified view of the facial recess region that is bounded by the facial nerve posteriorly and the chorda tympani anteriorly. The width of the facial recess may be as small as 2.4 $\mathrm{mm}$ [2] indicating that careful drilling is required to avoid damage to any structures. Average time for this traditional approach is 171 minutes [3].

To reduce the invasiveness and the surgery time, a minimallyinvasive surgical technique called "percutaneous cochlear implantation” (PCI) has been developed. PCI involves drilling a linear path from the skull to the cochlea through the facial recess avoiding damage to the surrounding vital anatomy. Due to the small target region (cochleostomy less than $1.5 \mathrm{~mm}$ in diameter)

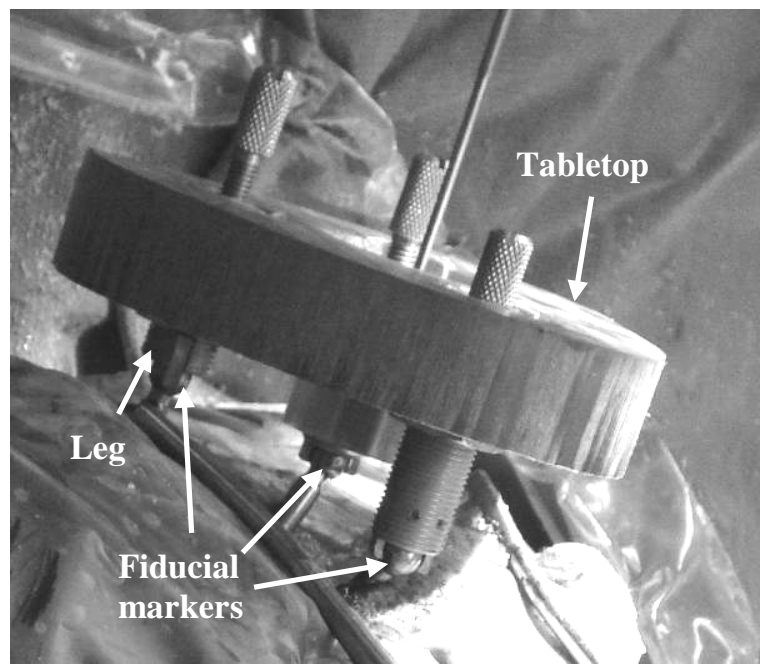

Figure 2. Microtable mounted on a patient for clinical validation of the PCI technique.

and the narrow region to drill through (facial recess as small as $2.4 \mathrm{~mm}$ wide), submillimetric accuracy is required for implementing PCI. To achieve this level of accuracy, customized microstereotactic frames that mount on boneimplanted markers are used to provide guidance and constrain a surgical drill along the planned path. This method is currently under clinical validation $[4,5]$.

We have tested two microstereotactic frames-the commercially available STarFix ${ }^{\mathrm{TM}}$ microTargeting ${ }^{\mathrm{TM}}$ Platform (FHC Inc., Bowdoin, ME) and a recently developed fixture called the Microtable ${ }^{\mathrm{TM}}$ —both of which achieve the accuracy necessary for PCI [4-8]. The STarFix ${ }^{\mathrm{TM}}$ platform, which is FDA-approved for use in deep-brain stimulation surgeries, is manufactured using rapid-prototyping technology at a centralized facility away from the hospital. Hence, there is a minimum of two days delay between the placement of markers and surgery causing inconvenience to both patient and surgeon. The Microtable was developed at Vanderbilt University to avoid this inconvenience. It can be constructed using a standard milling machine within few minutes [5]. The Microtable consists of a tabletop with three legs each of which mounts on a fiducial marker. The tabletop contains three holes to accommodate the three legs and a fourth hole, called the "target hole", to aim the surgical tool along the desired trajectory. For a given surgical procedure, the microtable is customized to mount on the fiducial markers with the tabletop perpendicular to the desired trajectory and with the top of the target hole $75 \mathrm{~mm}$ away from the cochlea. The desired leg lengths are selected and all the holes are countersunk to complete the custom design. Figure 2 shows the Microtable mounted on a patient during a clinical validation [5].

When the Microtable is used in conjunction with an intraoperative CT scanner, the whole PCI procedure can be performed inside the operating room in one setting. This technique has the potential to reduce the average surgical time to about 60 minutes. In this paper, we report a cadaveric study that demonstrates the insertion of electrode array into the cochlea using the PCI technique.

\section{METHODS}

We demonstrate on cadaveric temporal bone specimens the complete process to implant the cochlear implant into the cochlea using the PCI technique. Institutional review board approval was not necessary for this cadaveric study.

A custom manual insertion tool [9] (Figure 3) was developed to insert electrodes into the cochlea using the advance off-stylet (AOS) technique. The AOS technique is used for inserting electrode arrays that are usually pre-

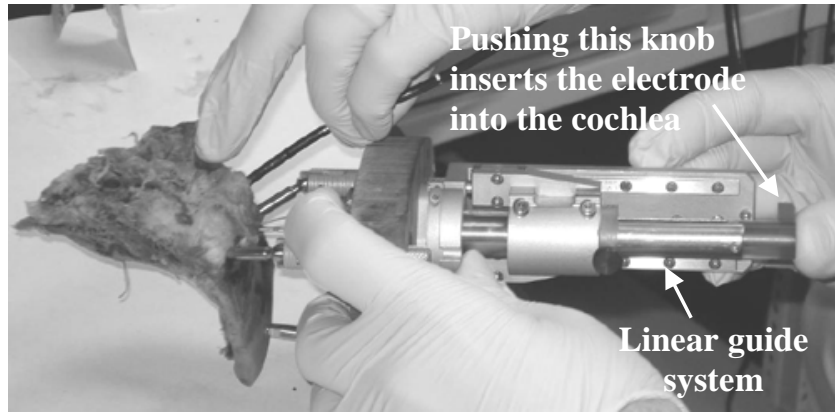

Figure 3. Insertion tool attached to the temporal bone specimen via Microtable to guide the electrode array into the cochlea through the drilled path. 

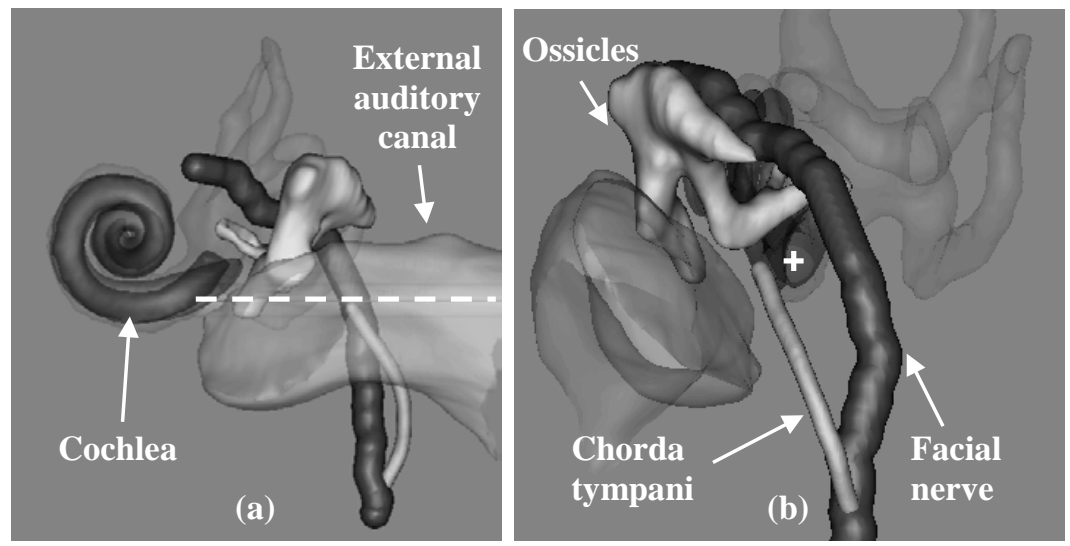

Figure 4. Trajectory planning. Significant structures of the ear are segmented, and a safe drill trajectory that targets the scala tympani within the cochlea is determined. (a) View from posterior to anterior. The white dashed line indicates the trajectory. (b) View as seen through the trajectory. The white cross indicates the target point in the cochlea.

curved to match the spiral shape of the cochlea, but held straight by inserting a stiff wire called the "stylet" into the central channel of the implant. During insertion into the cochlea, the electrode array and the stylet are inserted together straight into the cochlea for a specified distance until the tip of the implant reaches the first turn of the cochlea. Then the stylet is held fast and the electrode is advanced off the stylet and into the cochlea. At this point, the electrode array takes a natural curved shape conforming to the shape of the cochlea. The manual insertion tool requires setting the distance for the straight insertion prior to beginning of the insertion. Once set, the insertion of the electrode array requires only pushing a control knob that is available on the top of the tool. The straight insertion followed by the AOS insertion is automatically executed by the tool as described in [9]. The insertion tool is attached to a linear guide that allows easy attachment of the tool to the Microtable and also accurate advancement of the tool along the drilled path.

For this cadaveric study, we followed the same procedure that would be performed for a clinical implementation of PCI. The steps involved in this procedure are as follows.

1. Acquisition of preoperative CT scan. A clinically-applicable temporal CT scan of the patient is obtained using a traditional multi-slice CT scanner prior to the surgery.

2. Preoperative trajectory planning. Before the surgery, significant structures of the ear such as the cochlea, the facial nerve, the chorda tympani, the labyrinth, the ossicles, and the external auditory canal are automatically segmented in the preoperative CT scan using the published methods $[10,11]$ and a safe drill trajectory is automatically determined that targets the scala tympani component of the cochlea and avoids damage to critical structures mainly the facial nerve [12]. Figure 4 shows an example of the results of segmentation and drill trajectory for a patient. It takes approximately three minutes for completing this step with an Intel Xeon $2.4 \mathrm{GHz}$ dual quad-core 64-bit machine with $10 \mathrm{~GB}$ random access memory. The segmentation results and the chosen drill path are confirmed by the surgeon prior to the surgery. If not satisfied with the segmentation results or the drill path, manual adjustments may be performed. If a safe trajectory cannot be identified, then it is confirmed that the PCI technique cannot be performed for that patient.

3. Implantation of fiducial markers. Three fiducial markers are boneimplanted surrounding the ear. An anchor made of titanium is screwed into the bone, and a stainless steel "extender", which is solid cylinder with a spherical tip, is screwed into the anchor. The sphere on the extender is used as a fiducial marker and also used to mount the Microtable on the patient. Figure 5 shows three markers attached to a

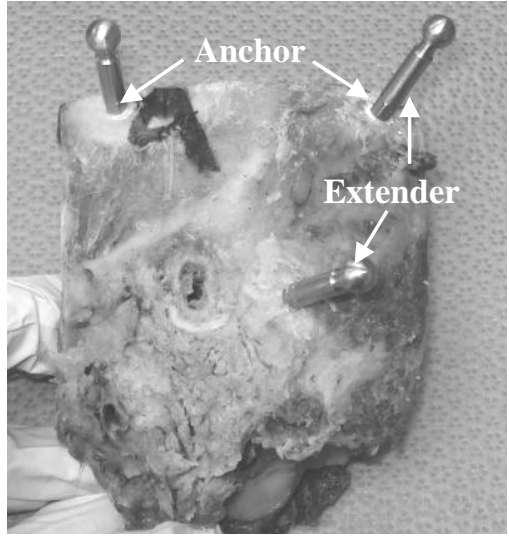

Figure 5. Three fiducial markers rigidly attached to the bone. An anchor is screwed into the bone, and an extender with the spherical end is attached to the anchor. 
temporal bone specimen. Based on prior clinical validation studies, average time to implant all the three fiducial markers is eight minutes.

4. Acquisition of intraoperative CT scan. A CT scan is then acquired covering the fiducial markers within the field of view. We use an xCAT ENT portable CT scanner (Xoran Technologies, Ann Arbor, MI) to acquire these intraoperative scans with a voxel size of $0.4 \mathrm{~mm}$ in all directions. This step requires approximately five minutes.

5. Intraoperative planning. The preoperative and the intraoperative CT

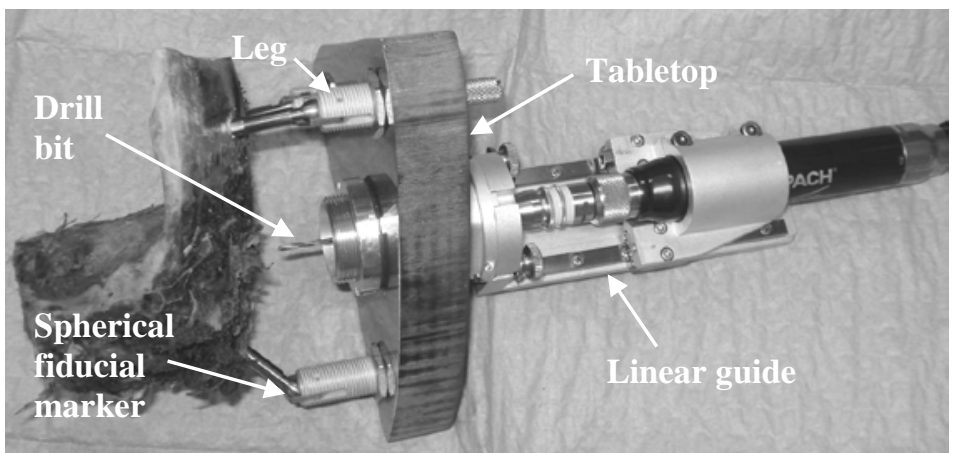

Figure 6. Microtable with the drill press assembly mounted on the temporal bone specimen. The Microtable is designed such that the drill bit travels along the desired drill path when pushed using the linear guide system.

scans are rigidly registered using the standard mutual-information method [5]. Via the resulting transformation, the segmentations and the desired drill trajectory are transformed from the preoperative scan to the intraoperative scan. The centers of the spherical fiducial markers are localized in the intraoperative scan. This step requires approximately four minutes.

6. Design and fabrication of the surgical guide. A Microtable is designed automatically with custom Matlab (Mathworks, Natick, MA) software using the location of the three fiducial markers and the trajectory as input such that the Microtable will mount, as shown in Figure 6, on the fiducial markers and constrain the drill to the planned drill path. The software outputs the G-code necessary as input to a computer-numeric-control (CNC) milling machine for fabrication of the tabletop and specifies lengths for the legs. Fabrication requires less than five minutes. An adaptor is then attached to the target hole of the tabletop that allows attachment of the linear guide of the drill press and the insertion tool to the Microtable, and legs of the specified lengths are attached to the corresponding holes in the tabletop. Attachment of the adaptor and legs requires approximately two minutes. A quality assurance check is then performed to confirm accurate fabrication of the Microtable. This check requires approximately one minute.

7. Drilling to the cochlea. A custom drill press is attached to the adaptor in the target hole of the Microtable, and the Microtable with drill press attached is mounted on the fiducial markers. Drilling is then performed as described in [6]. Figure 6 shows the Microtable with the drill press mounted on a temporal bone specimen. The path of the drill bit is defined by the Microtable. After drilling, the drill press is removed from the Microtable.

8. Insertion of the electrode. A stop on the insertion tool is adjusted to set the distance for straight insertion before beginning the AOS insertion. This distance is manually determined on the preoperative CT scan. The electrode array is then loaded to the insertion tool following which the linear guide of the insertion tool (Figure 3) is attached to the adaptor in the target hole of the Microtable. The insertion tool is pushed along the linear guide until the stop restricts further forward movement. At this point, the tip of the insertion tool is at the desired target location. Then using a control knob on the tool, the electrode array is pushed into the cochlea using the AOS technique.

\section{RESULTS}

PCI was performed on three cadaveric temporal bone specimens. All the steps described in the Methods section were performed in sequence for each cadaveric specimen. The preoperative CT scans were acquired using a 16-slice Philips Mx8000 IDT 16 CT scanner that provided images with voxel size of 0.25 -by- 0.25 -by- $0.4 \mathrm{~mm}$. The segmentation results and the planned trajectory of the automatic trajectory planning were found satisfactory for all specimens, and no manual adjustments were found necessary. Fiducial markers were bone-implanted on the specimen, one at each of the following positions: the mastoid tip, the suprahelical region, and the region posterior to the sigmoid sinus, as shown in Figure 5. A custom Microtable was made for each specimen to enable drilling to the cochlea and insertion of electrode array into the 

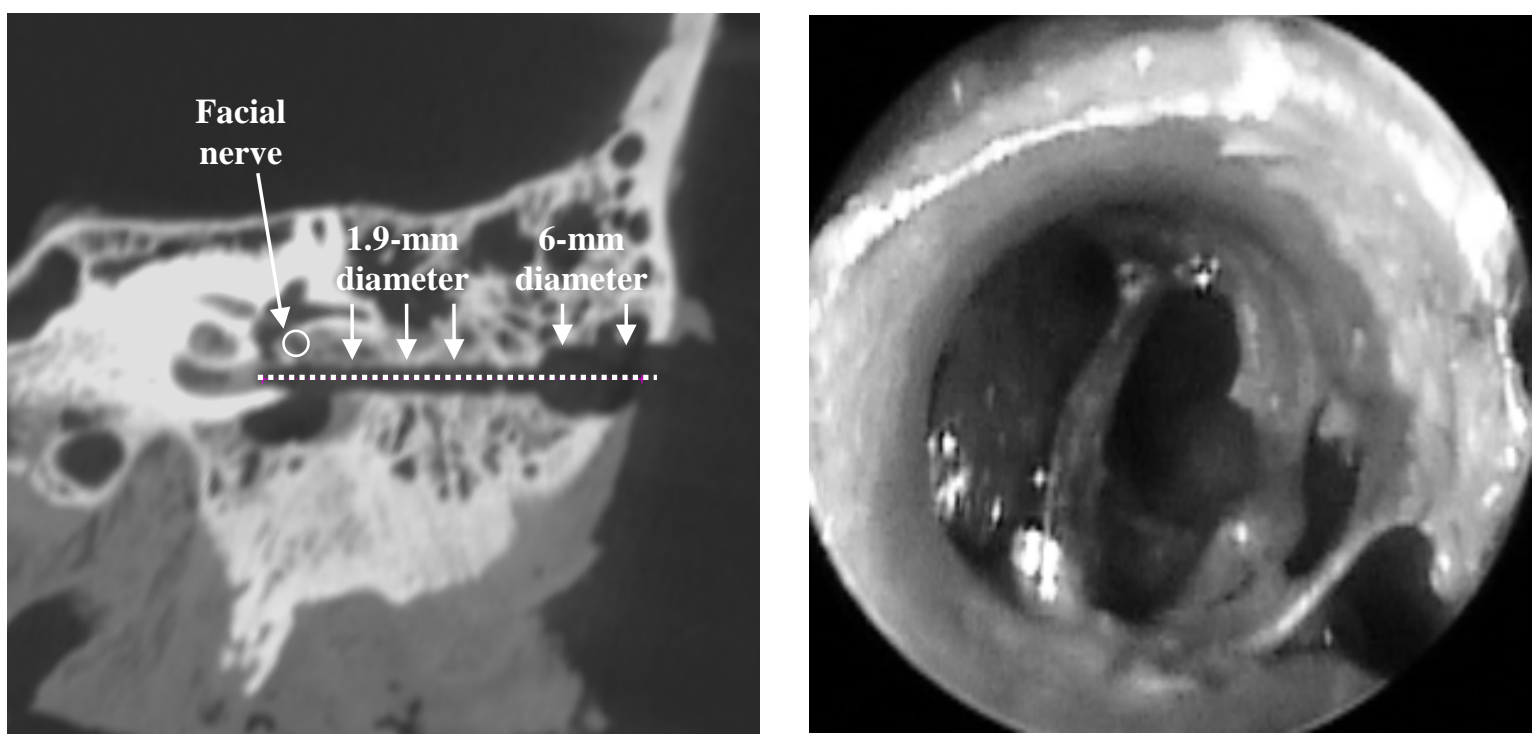

Figure 7. Confirmation of the drilled path. (a) Post-drilling CT scan showing the drilled path achieved using the PCI technique. The white dotted line shows the axis of the desired trajectory. (b) Endoscopic view of the target achieved after the drilling.

cochlea along the desired path.

Drilling was performed as described in [8] using a $6 \mathrm{~mm}$ twist drill bit from the lateral skull toward the target until the drill was $20 \mathrm{~mm}$ from the target. A $1.9 \mathrm{~mm}$ twist drill bit was used for the remaining $20 \mathrm{~mm}$ to the target. Drill bushing adaptors were used (not shown in Figure 6) to restrict the movement of the drill bit during drilling. It required less than five minutes to complete the drilling once the Microtable was ready. A CT scan was acquired after drilling to determine whether the desired trajectory was achieved. In all the specimens, the drill reached the cochlea without damaging the facial nerve. Figure 7(a) provides a screenshot of a post-drill CT scan showing the drilled path to the cochlea and the planned path (white dotted line). It can be seen that the drilling was accurately performed along the desired path and that
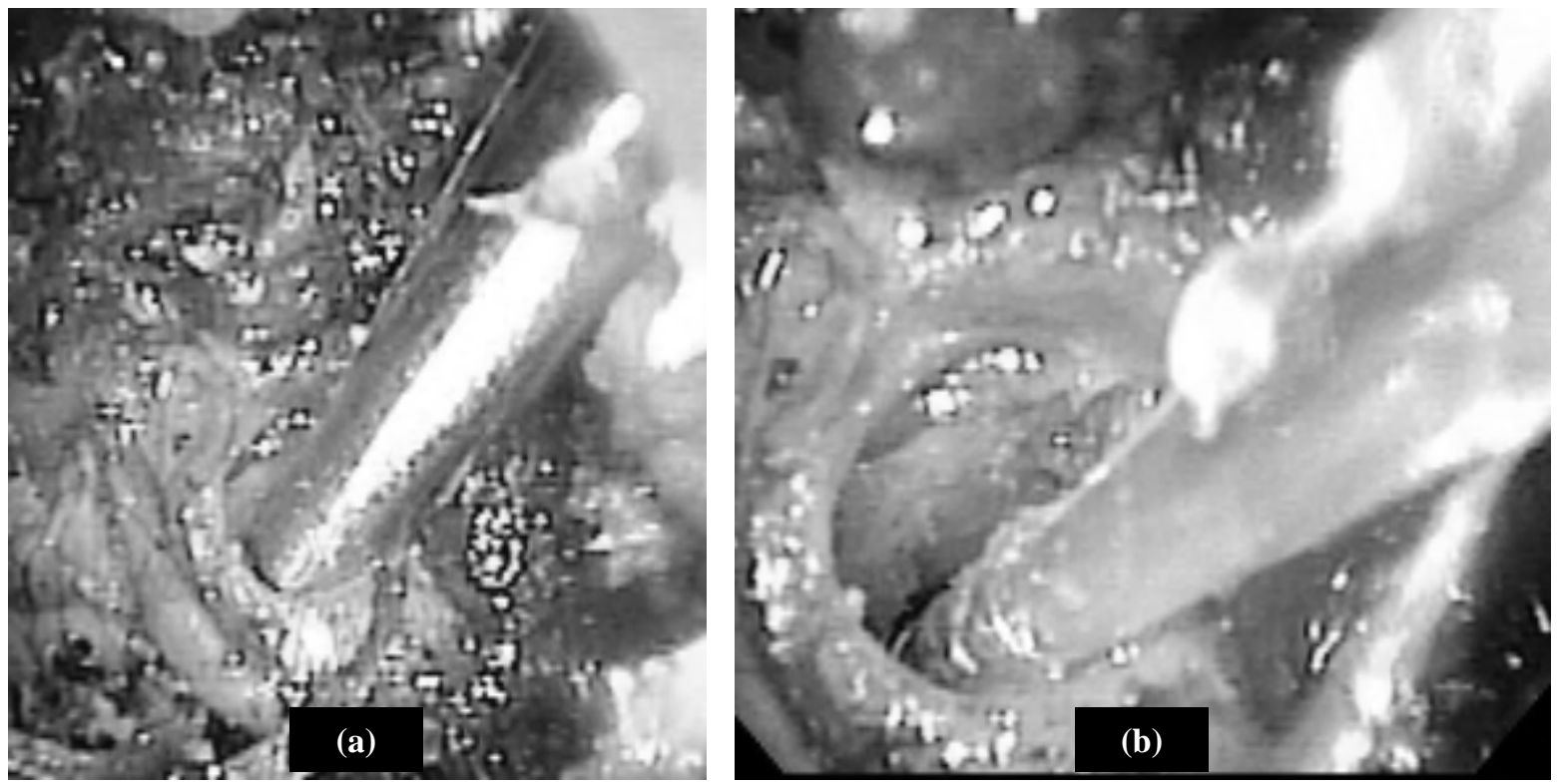

Figure 8. Endoscope view through the ear canal to confirm the insertion. (a) View after the insertion tool was inserted into the drilled hole and before deployment of the electrode array showing that the tip of the insertion tool is aimed into the cochlea. (b) View after the insertion tool was removed showing that the electrode array is inside the cochlea. 
the facial nerve (highlighted by a white circle) was not damaged in the process. Confirmation was also performed by inserting an endoscope through the drilled hole to visualize the cochlea. Figure 7(b) shows the endoscope view through the drilled hole of a specimen confirming the successful opening of the cochlea.

Insertion was then performed using the Microtable and the manual insertion tool (Figure 3). Practice Nucleus Contour Advance electrodes (Cochlear Corporation, Lane Cove, New South Wales, Australia), which failed quality control but were otherwise identical to clinically-used electrodes were used in this study. The insertion tool with the electrode array loaded was attached to the Microtable and advanced into the drilled hole until the stop on the linear guide prohibited further advancement. During the insertion in each specimen, an endoscope view through the ear canal was continuously monitored to verify proper insertion. It was confirmed using the endoscope that the tool tip was in the cochlea. Figure 8(a) shows the placement of the tool tip for insertion. The electrode was then inserted by pushing a control knob on the insertion tool. Insertion of the electrode after the tool has

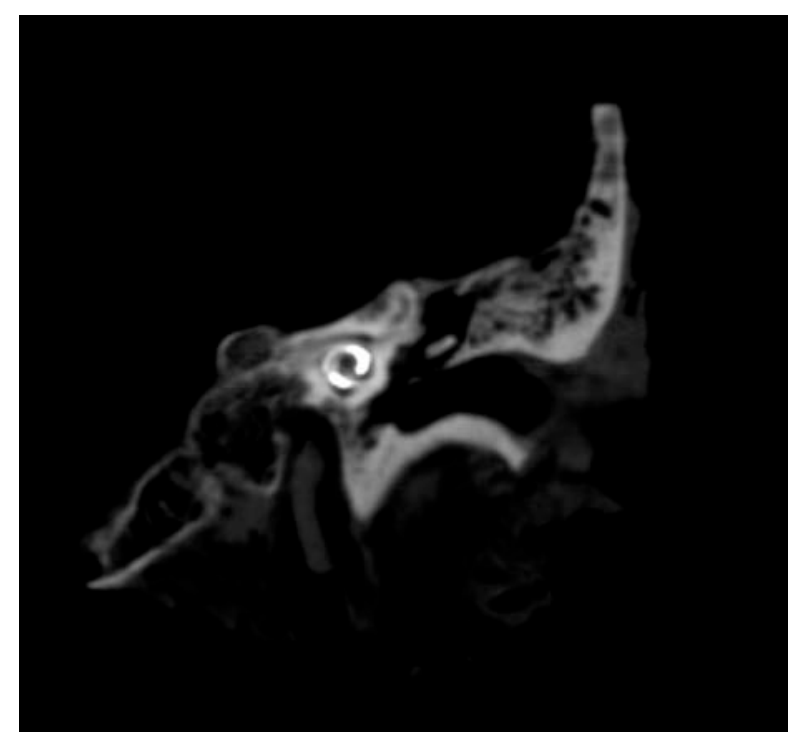

Figure 9. Post-insertion CT scan showing the results of PCI. The electrode array (white) is within the cochlea as desired. been loaded with the electrode required less than two minutes. After insertion, the insertion tool and the Microtable were removed from the specimen leaving the electrode array inside the cochlea. Figure 8(b) shows the endoscope view confirming that the electrode is inside the cochlea. A CT scan was acquired after insertion of the electrode to analyze the quality of the insertion. In all the specimens, the insertion was satisfactory with the electrode array inside the cochlea as desired. Figure 9 shows a screenshot of a postinsertion CT scan confirming the correct placement of the electrode array inside the cochlea.

\section{DISCUSSION}

PCI was performed on three $(n=3)$ cadaveric temporal bone specimens using custom Microtables that mount on boneimplanted markers. Drilling to the cochlea was accomplished successfully along the desired path without damage to the facial nerve followed by successful insertion of the electrode into the cochlea.

Traditional cochlear implantation surgery involves a wide surgical exposure to clearly identify anatomic landmarks, thereby avoiding damage to critical structures [1]. Compared to this traditional approach, PCI involves drilling a single path from the lateral skull to the cochlea via a path that is chosen from CT scans to avoid critical structures. PCI requires making only three small incisions (less than $1 \mathrm{~cm}$ wide) to attach fiducial markers to the skull and a fourth incision about $2 \mathrm{~cm}$ wide for the drill path and to insert the internal receiver underneath the skin. Because the drill path travels close to critical structures, it is crucial to accurately guide the drill and insertion tool along the desired path. To achieve this high accuracy, we depend on a system with a low probability of error [14] that is based on bone-implanted fiducial markers and the custom microstereotactic frame that rigidly attaches to these markers. A custom drill press and insertion tool were developed that each rigidly attach to the Microtable to accurately perform the drilling and insertion, respectively.

With the availability of a portable CT scanner and the ability to fabricate custom Microtable within minutes, we hypothesize that it is possible to consistently perform the entire PCI within 60 minutes. Portable CT scanners, which cost about \$250,000, are becoming increasingly popular in hospitals to use with patients in intensive care units [15]. It might soon become standard equipment in hospitals. The other machine required is a CNC machine, which costs about $\$ 15,000$ for a miniaturized version that can be situated close to the operating room to avoid delay in the transportation of the Microtable to the surgical field. With the significant time savings of PCI compared to the traditional surgery, this capital expense could be quickly offset. . 


\section{CONCLUSION}

In a cadaveric model, we have demonstrated the implementation of a minimally-invasive technique for cochlear implantation called "percutaneous cochlear implantation" (PCI) using customized microstereotactic frames. Customized Microtables, which are microstereotactic frames that can be manufactured in less than five minutes, were used to guide the drill and the insertion tool along the desired path. Drilling to the cochlea and insertion of an electrode array into the cochlea were successfully performed without damage to the facial nerve. The results provide encouragement to extend this technique to clinical implementation.

\section{ACKNOWLEDGEMENTS}

The project described was supported by award number R01DC008408 from the National Institute on Deafness and Other Communication Disorders. The content is solely the responsibility of the authors and does not necessarily represent the official views of the National Institute on Deafness and Other Communication Disorders or the National Institutes of Health. The authors would like to thank Dr. Thomas Cleveland for his immense help with the acquisition of the endoscope images.

\section{REFERENCES}

1. W. House, "Surgical considerations in cochlear implantation,” Ann Otol Rhinol Laryngol Supp vol. 91, no. 2 Pt 3, pp. 15-20, 1982.

2. Su W, Marion MS, Hinojosa R, Matz GJ. "Anatomical measurements of the cochlear aqueduct, round window membrane, round window niche, and facial recess," Laryngoscope 1982;92:483-6.

3. O. Majdani, T. A. Schuman, D. S. Haynes et al., "Time of cochlear implant surgery in academic settings," Otolaryngol Head Neck Surg, vol. 142, no. 2, pp. 254-9, Feb, 2010.

4. R. F. Labadie, J. H. Noble, B. M. Dawant et al., "Clinical validation of percutaneous cochlear implant surgery: initial report,” Laryngoscope, vol. 118, no. 6, pp. 1031-9, Jun, 2008.

5. R. F. Labadie, R. Balachandran, J. Mitchell et al., "Clinical Validation Study of Percutaneous Cochlear Access Using Patient Customized Micro-Stereotactic Frames,” Otol Neurotol, vol. 31, no. 1, pp. 94-99, 2010.

6. R Balachandran, J Mitchell, B. M. Dawant, J. M. Fitzpatrick, “Accuracy evaluation of MicroTargeting ${ }^{\mathrm{TM}}$ platforms for deep-brain stimulation using virtual targets," IEEE Transactions on Biomedical Engineering, 56(1), 37-44, Jan 2009.

7. R. F. Labadie, J. Mitchell, R. Balachandran et al., "Customized, rapid-production microstereotactic table for surgical targeting: description of concept and in vitro validation,” Int J CARS, vol. 4, pp. 273-280, 2009.

8. R. Balachandran, J. Mitchell, G. Blachon et al., "Percutaneous Cochlear Implant Drilling via Customized Frames: an in vitro study ” Otolaryngol Head Neck Surg, vol. 142, no. 3, pp. 421-426, 2010.

9. D. Schurzig, Z. W. Smith, D. C. Rucker et al., "A Manual Insertion Mechanism for Percutaneous Cochlear Implantation,” in Design of Medical Devices Conference, Minneapolis, MN, USA, 2010, pp. 3901.

10. J. H. Noble, F. M. Warren, R. F. Labadie et al., "Automatic segmentation of the facial nerve and chorda tympani in CT images using spatially dependent feature values,” Med Phys, vol. 35, no. 12, pp. 5375-84, Dec, 2008.

11. J. H. Noble, B. M. Dawant, F. M. Warren et al., "Automatic identification and 3D rendering of temporal bone anatomy,” Otol Neurotol, vol. 30, no. 4, pp. 436-42, Jun, 2009.

12. J. H. Noble, F. M. Warren, R. F. Labadie et al., "Determination of drill paths for percutaneous cochlear access accounting for target positioning error," Progress in Biomedical Optics and Imaging - Proceedings of SPIE, vol. 6509, pp. 650925.1-650925.10, 2007.

13. Maes F, Collignon A, Vandermeulen D, et al. «Multimodality image registration by maximization of mutual information.” IEEE Trans Med Imaging1997;16:187-98.

14. Fitzpatrick JM, West JB. "The distribution of target registration error in rigid-body point-based registration." IEEE Trans Med Imaging 2001; 20:917-27.

15. Peace K, Wilensky EM, Frangos S, MacMurtrie E, Shields E, Hujcs M, Levine J, Kofke A, Yang W, Le Roux PD, "The use of a portable head CT scanner in the intensive care unit," J. Neurosci Nurs. 2010 Apr;42(2):10916. 